\title{
Circulating branched-chain amino acid concentrations are associated with skeletal muscle mass and thyroid function in young Japanese men
}

Katsuhiko Tsunekawa ( $\nabla$ ktsune@gunma-u.ac.jp )

Gunma University Graduate School of Medicine https://orcid.org/0000-0002-6119-4158

Ryutaro Matsumoto

Nippon Sport Science University

Kazumi Ushiki

Gunma University Graduate School of Medicine

Larasati Martha

Gunma University Graduate School of Medicine

Yoshifumi Shoho

Ikuei University

Yoshimaro Yanagawa

Ikuei University

Hirotaka Ishigaki

Gunma Paz University

Akihiro Yoshida

Gunma University Graduate School of Medicine

Osamu Araki

Gunma University Graduate School of Medicine

Kiyomi Nakajima

Gunma University Graduate School of Medicine

Takao Kimura

Gunma University Graduate School of Medicine

Masami Murakami

Gunma University Graduate School of Medicine

Research article

Keywords: Skeletal muscle mass, Branched-chain amino acid (BCAA), Albumin, Thyroid hormone

Posted Date: April 6th, 2020

DOI: https://doi.org/10.21203/rs.3.rs-20621/v1 
License: (c) (i) This work is licensed under a Creative Commons Attribution 4.0 International License. Read Full License 


\section{Abstract}

Background Few nutritional markers reflect the hypermetabolic state of athletes with high levels of skeletal muscle. Although branched-chain amino acids (BCAA) play crucial roles in protein metabolism in skeletal muscle, the relation between circulating BCAA concentration and skeletal muscle mass has not been fully understood. The aim of this study is to evaluate the association between skeletal muscle mass and serum BCAA to tyrosine ratio (BTR), a convenient indicator of circulating BCAA, in young Japanese men including wrestling athletes with high skeletal muscle mass.

Methods The study enrolled 111 young Japanese men: 70 wrestling athletes and 41 controls. None were taking medications or undergoing extreme dietary restriction or an intense exercise regimen. Each participant's body composition, serum concentrations of albumin and rapid turnover proteins, BTR, and thyroid function were assessed.

Results Compared to the controls, the athletes had significantly higher weight-adjusted skeletal muscle mass (SMM) $(p<0.001)$ and skeletal muscle index (SMI) $(p<0.001)$, and lower serum albumin concentration $(p<0.001)$ and BTR $(p<0.001)$. Kruskal-Wallis tests with Bonferroni multiple comparison tests showed that serum albumin concentration and BTR were significantly lower in the participants with higher SMM and SMI. Spearman's correlation analyses showed that SMM and SMI were inversely correlated with serum albumin concentration (SMM, $\rho=-0.315, p=0.001$; SMI, $\rho=-0.511, p<0.001$ ) and BTR (SMM, $\rho=-0.424, p<0.001 ; S M I, \rho=-0.511, p<0.001)$. Serum albumin concentration was positively correlated with BTR $(\rho=0.438, p<0.001)$, transthyretin $(\rho=0.281, p=0.003)$ and transferrin $(\rho$ $=0.299, p=0.001)$, but BTR was not correlated with other nutritional indicators. Serum concentration of free 3,5,3'-triiodothyronine was inversely correlated with BTR $(\rho=-0.308, p=0.001)$, but not with serum albumin or skeletal muscle mass.

Conclusions These results suggest that the consumption of circulating BCAAs increases with increased skeletal muscle mass and is facilitated by thyroid hormones independent of skeletal muscle mass. Serum BTR may be a useful biomarker to assess the hypermetabolic state of athletes.

\section{Background}

Because of their branched structure, the essential amino acids valine, leucine, and isoleucine are collectively referred to as the branched-chain amino acids (BCAAs). These amino acids play crucial roles in skeletal muscle [1], not only as a major component of proteins, but also as an energy source, especially during exercise $[1,2]$. BCAAs are also involved in the regulation of protein metabolism in skeletal muscle cells; for example, leucine activates mammalian target of rapamycin complex 1 (mTORC1), which stimulates protein synthesis and suppresses proteolysis by autophagy [3]. This activation of mTORC1 requires a high concentration of circulating leucine to be maintained [4].

Sarcopenia (i.e., a decrease in skeletal muscle mass) is a serious clinical problem associated with poorer prognosis in various diseases $[5,6]$. Studies have reported a relationship between skeletal muscle mass 
and the concentration of circulating BCAAs in several diseases $[7,8]$. Patients with chronic heart failure showed lower serum BCAA concentrations and Fisher's ratios than controls, with positive correlations between the values of their skeletal muscle index (SMI), defined as skeletal muscle mass divided by the squared height, and their BCAA concentrations and Fisher's ratios [7]. In patients with chronic liver diseases, it was observed that lower BCAA to tyrosine ratios (BTRs) were associated with decreased skeletal muscle mass [8].

Through training, sports athletes increase their skeletal muscle mass for more effective energy use and improved competitive performance. Exercise induces an increase in whole-body energy expenditure and a prompt decrease in circulating BCAAs [9]. Several studies have reported that BCAA supplementation reduces muscle damage and protein breakdown during exercise [10-12]. In circulating lipid metabolism, lipoprotein lipase (LPL) plays a crucial role in triglyceride (TG)-rich lipoprotein hydrolysis [13]. LPL is highly expressed and synthesized in skeletal muscle tissues to use fatty acids for energy and translocated to the capillary lumen by glycosylphosphatidylinositol anchored high-density lipoprotein binding protein 1 (GPIHBP1), which has a vital role in LPL lipolytic processing [14]. We previously reported that wrestling athletes with high levels of skeletal muscle had high concentrations of LPL and GPIHBP1, and that increasing skeletal muscle mass improved effective energy use by promoting the hydrolysis of TG-rich lipoproteins [15]. However, the relationship between skeletal muscle mass and protein metabolism, including circulating BCAAs, has not been fully understood.

Thyroid hormones also play a vital role in energy metabolism in skeletal muscle [16]. They increase oxygen consumption and resting metabolic rate through increased mitochondrial activity related to the stimulation of mitochondrial enzymes and uncoupling protein 3 [17]. Thyroid hormones also promote skeletal muscle differentiation and induce the transition from slow to fast fibers by suppressing of Myh7 gene expression and through the stimulation of Myh1, Myh2, and Myh4 expression [17]. However, the role of thyroid hormones in amino acid metabolism in skeletal muscle, especially that of BCAAs, has not been elucidated, and the relationships between serum concentrations of free thyroid hormones and BCAAs in athletes with regular high energy consumption are not known.

Nutritional indicators, such as levels of albumin and rapid turnover proteins, are often used as blood biomarkers for assessing the condition of athletes to help their good performance. However, few markers reflect the hypermetabolic state of athletes. If the relationship between skeletal muscle mass and the concentration of circulating BCAAs can be clarified in athletes, it may be possible to use BCAA concentration as a biomarker of the hypermetabolic state. In addition, clarification of the relationship between concentrations of circulating BCAAs and thyroid function tests could help elucidation of the novel mechanisms of BCAA metabolism via thyroid hormones in skeletal muscle. Concentrations of circulating BCAAs are typically detected by amino acid analysis using liquid chromatography-mass spectrometry, which is cumbersome and not widely available [18]. In contrast, serum BTR, which provides a simple indication of circulating BCAA concentration and Fisher's ratio, can be measured conveniently. 
The aim of this study was to investigate the associations between serum BTR, skeletal muscle mass, and thyroid function in young Japanese men, including athletes with high skeletal muscle mass, and to compare BTR to other nutritional indicators.

\section{Materials And Methods Participants}

This study was a subanalysis of a previous cross-sectional study [15]. In brief, we enrolled 111 young, healthy Japanese: 70 elite amateur wrestlers and 41 college students who did not engage in habitual hard exercise. None were taking medications for metabolic diseases. All the participants provided written informed consent before being included in the study. The study was approved by the ethics committee of Gunma University Graduate School of Medicine (approval no. 13-36).

\section{Physical Examinations}

The athletes were assessed at a time when they were not under any dietary restriction and were not undergoing intense training for a tournament. Blood samples were collected and physical examinations were performed in the morning after a 12-h fast period without exercise. A bioimpedance instrument (InBody 430; InBody Japan, Tokyo, Japan) was used to measure body weight, fat mass, and skeletal muscle mass, with the participant in the standing position. The following indices were calculated: body mass index (BMI) as weight/height squared; fat mass index (FMI) as fat mass/height squared; skeletal muscle index (SMI) as skeletal muscle mass/height squared; \% weight-adjusted fat mass (FM) as fat mass/weight; and \% weight-adjusted skeletal muscle mass (SMM) as skeletal muscle mass/weight (\%).

\section{Thyroid Function, Serum Concentrations of Albumin and Rapid Turnover Proteins, and BTR}

With the participant sitting, blood samples were collected from an antecubital vein using 23-G needles. The serum samples were separated by centrifugation $(1,500 \times \mathrm{g})$ at $4{ }^{\circ} \mathrm{C}$ for 10 min and were stored at $80^{\circ} \mathrm{C}$ until analysis. A LABOSPECT 008 automatic analyzer (Hitachi, Tokyo, Japan) was used to measure serum albumin concentrations using the modified bromocresol purple method, serum retinol binding protein (RBP) concentrations using latex turbidimetric immunoassays, and serum transthyretin and transferrin concentrations using turbidimetric immunoassays. A chemiluminescent microparticle immunoassay on an Abbott ARCHITECT i2000SR Immunoassay Analyzer (Abbott Laboratories, Abbott Park, IL, USA) was used to analyze serum concentrations of free 3,5,3'-triiodothyronine $\left(\mathrm{FT}_{3}\right)$, free thyroxine $\left(\mathrm{FT}_{4}\right)$, and thyrotropin (TSH). Serum BTR was measured by the enzymatic method by LSI Medience Co. (Tokyo, Japan).

\section{Statistical Analysis}

Since most of the variables were not normally distributed, the data are expressed as median values with 25th-75th percentiles. Mann-Whitney U tests were used, as appropriate, to identify statistically significant 
differences between the two study groups. Kruskal-Wallis tests with Bonferroni multiple comparison tests were performed to compare the two groups classified by quartile. Spearman's correlation analyses were performed to evaluate the relationships between serum albumin concentrations or BTR and the clinical parameters. Differences and correlations were considered significant when $p<0.05$. SPSS Statistics version 25.0 (IBM Corp., Armonk, NY, USA) was used for the statistical analyses.

\section{Results}

\section{Clinical Characteristics of the Participants}

Table 1 presents the clinical characteristics for the two participant groups. Compared with the control group, the athlete group had significantly higher body weights $(p=0.012), B M I(p<0.001), S M M(p<$ $0.001)$, and SMI $(p<0.001)$, and lower FM $(p<0.001)$ and FMI $(p=0.024)$, as reported previously [15]. The serum concentration analyses showed that the athlete group had significantly higher serum concentrations of RBP $(p=0.009)$ and lower serum concentrations of albumin $(p<0.001)$ and BTR $(p<$ 0.001). Serum concentrations of transferrin tended to be lower in the athlete group, although the difference was not statistically significant $(p=0.071)$. There were no differences in thyroid function tests between the groups, as reported previously [15].

\section{Skeletal Muscle Mass, Serum Albumin Concentrations, and BTR}

Figures 1 and 2 show the associations between skeletal muscle mass (expressed as SMM or SMI), serum albumin concentrations, and BTR for all the participants. For this analysis, the participants were divided into four groups classified by quartile for SMM or SMI. In each case, there were significant differences among the quartiles (SMM: serum albumin concentration, $p=0.006$; BTR, $p=0.001$; SMl: serum albumin concentration, $p<0.001$; BTR, $p<0.001$; Kruskal-Wallis tests). In Bonferroni multiple comparison tests, the serum albumin concentrations were significantly lower in Quartiles 3 and 4 than in Quartile 2 for SMM, and significantly lower in Quartiles 2, 3, and 4 than in Quartile 1 for SMI (Fig. 1A). Similarly, BTR was significantly lower in Quartiles 3 and 4 than in Quartile 1 for SMM, and significantly lower in Quartile 4 than in Quartile 2; and it was significantly lower in Quartile 3 and 4 than in Quartile 1 for SMI (Fig. 1B). The Spearman's correlation analyses showed that serum albumin concentration was inversely correlated with SMM $(\rho=-0.315, p=0.001)$ and SMI $(\rho=-0.511, p<0.001)$ (Fig. 2A), and BTR was inversely correlated with SMM $(\rho=-0.424, p<0.001)$ and SMI $(\rho=-0.436, p<0.001)$ (Fig. 2B).

\section{Correlations Between Serum Albumin Concentrations or BTR and Clinical Variables}

Table 2 and Fig. 3 show the correlations between serum albumin concentration or BTR and clinical variables for all the participants. Serum albumin concentration was positively correlated with FM $(\rho=$ $0.240, p=0.011)$, transthyretin $(\rho=0.281, p=0.003)$, transferrin $(\rho=0.299, p=0.001)$, and BTR $(\rho=0.438$, 
$p<0.001$; Fig. 3A), and inversely correlated with body weight $(\rho=-0.213, p=0.025)$ and $\operatorname{BMI}(\rho=-0.370$, $p<0.001$ ), as well as with SMM and SMI, as noted in the previous section. There were no significant correlations between serum albumin concentration and the thyroid function tests. BTR was positively correlated with FM $(\rho=0.334, p<0.001)$ and FMI $(\rho=0.238, p=0.012)$, and inversely correlated with body weight $(\rho=-0.214, p=0.024)$, BMI $(\rho=-0.217, p=0.022)$, SMM, SMI, and FT $3(\rho=-0.308, p=0.001$; Fig. 3 C). $\mathrm{FT}_{3}$ was not significantly correlated with SMM or SMI (SMM, $\rho=0.060, p=0.531 ; \mathrm{SMI}, \rho=0.114$, $p=0.234)$.

\section{Discussion}

This study investigated associations between skeletal muscle mass and nutritional indicators, including serum concentrations of albumin, rapid turnover proteins, and BCAAs, as well as the associations between these indicators and thyroid function tests, in young Japanese men. The wrestling athletes with high levels of skeletal muscle mass had significantly lower serum albumin concentrations and BTRs than the control participants. In all the participants, serum albumin concentration and BTR were inversely correlated with SMM and SMI. Serum $\mathrm{FT}_{3}$ concentration was inversely correlated with serum BTR, but not with skeletal muscle mass or the other nutritional indicators, including albumin.

Previous reports have compared concentrations of circulating albumin and rapid turnover proteins between athletes and controls. Serum albumin concentrations tended to be lower in male professional cyclists and skiers than in healthy controls [19]. Among athletes, rowers had lower plasma albumin concentrations than intermittent fasted athletes, such as Ramadan-fasted runners and boxers, and lightweight rowers had lower albumin concentrations than heavyweight rowers [20]. Serum transthyretin concentrations were higher in elite marathon runners than in controls, but there were no differences in RBP or transferrin concentrations between these groups [21]. These indicators are influenced by many exercise-related factors, including dehydration and inflammation, so no definite findings have been obtained for these indicators as markers for assessing the conditions of athletes who are undergoing continuous training. In addition, the relationship between skeletal muscle mass and these indicators remains unclear. For example, the relationship between levels of circulating BCAAs and the skeletal muscle mass of athletes in a resting state has not been investigated, although the effects of BCAA supplementation on exercise have been extensively studied in athletes [10-12].

In the present study, serum albumin concentration and BTR were lower in the wrestling athletes than in the controls and were inversely correlated with skeletal muscle mass. Although serum albumin concentration was positively correlated with levels of rapid turnover proteins, there was no significant correlation between serum BTR and rapid turnover proteins. These results suggest that, even at rest, albumin and BCAAs may be used as sources of protein in skeletal muscles to a greater extent than other marker proteins. The serum albumin concentration also reflects liver synthesis ability, but the serum BTR does not. Therefore, the serum BTR is considered to more strongly reflect the state of increased energy consumption within skeletal muscles, especially in athletes. In contrast to the correlations found in the present study, circulating BCAA levels have been reported to decrease with the decreases in skeletal 
muscle mass in patients with chronic liver diseases [8] and chronic heart failure [7]. In those studies, the diseases may have led to increased BCAA catabolism or insufficient BCAA intake, resulting in decreased concentrations of circulating BCAAs, which in turn may lead to a reduction in skeletal muscle mass. Future studies are needed to determine whether the concentration of circulating BCAAs reflects energy expenditure in skeletal muscle, through a detailed assessment of BCAA intake and measurement of other biomarkers of skeletal muscle mass and function.

Muscle cross-sectional area has been reported to be lower in elderly subclinical hypothyroid patients than in age-matched euthyroid controls [22]. A recent study reported that serum $\mathrm{FT}_{3}$ concentrations were positively correlated with appendicular skeletal muscle mass, handgrip strength, and the results of a short physical performance battery in elderly Chinese euthyroid subjects [23]. In that study, the $\mathrm{FT}_{3}$ concentrations were especially low in subjects with sarcopenia. Consistent with those results, we did not observe a correlation between skeletal muscle mass and thyroid function tests although, unlike the previous studies, our study involved young participants with adequate skeletal muscle mass. However, we observed an inverse correlation between serum $\mathrm{FT}_{3}$ concentration and serum BTR. A study of rats with hyperthyroidism showed that leucine supplementation improved their swimming performance [24]; this suggests that thyroid hormones increase BCAA metabolism in skeletal muscle, which may be relevant to our results. Further studies are needed to elucidate the mechanisms of BCAA metabolism in skeletal muscle involving thyroid hormones.

This study had several limitations. One was its relatively small sample size. During the enrollment, we specifically recruited elite wrestling athletes with high skeletal muscle mass at a time when they were not undergoing intense training or extreme restriction of nutrition and water intake for a tournament. Another limitation is that the participants' diets prior to the sample collection were not fully standardized. It has been reported that plasma BCAA concentrations in healthy male students increase immediately after BCAA ingestion, peak at $30 \mathrm{~min}$, and gradually decrease to the initial level by $180 \mathrm{~min}$ after ingestion [25]. In the present study, nutritional status, including BCAA metabolism, in the athletes and the controls who did not exercise and have breakfast from wake-up to sample collection after overnight fasting may have been at baseline without any immediate influence. Further studies are needed to confirm our hypothesis through investigations of athlete with various types of specialized skeletal muscle function, such as instantaneous power or endurance strength.

\section{Conclusions}

This study showed that serum albumin concentrations and BTRs in young Japanese men were significantly lower in wrestling athletes with high levels of skeletal muscle than in controls, and that they were inversely correlated with skeletal muscle mass. These results suggest that an increase in skeletal muscle mass results in enhanced consumptions of circulating albumin and BCAA to maintain the muscle mass. The metabolism of circulating BCAAs may also be increased by thyroid hormones independent of skeletal muscle mass. It may be possible to use BTR as a biomarker of hypermetabolic state in athletes. 


\section{List Of Abbreviations}

BCAA: branched-chain amino acid; BMI: body mass index; BTR: branched-chain amino acid to tyrosine ratio; FM: weight-adjusted fat mass; $F M l$ : fat mass index; $\mathrm{FT}_{3}$ : free 3,5,3'-triiodothyronine; $\mathrm{FT}_{4}$ : free thyroxine; GPIHBP1: glycosylphosphatidylinositol anchored high-density lipoprotein binding protein 1; LPL: lipoprotein lipase; mTORC1: mammalian target of rapamycin complex 1; RBP: retinol binding protein; SMI: skeletal muscle index; SMM: weight-adjusted skeletal muscle mass; TG: triglyceride; TSH: thyrotropin

\section{Declarations}

\section{Ethics approval and consent to participate}

Written informed consent was obtained from all participants. This study was approved by the ethics committee of Gunma University Graduate School of Medicine (Approval number 13-36).

\section{Consent for publication}

Not applicable.

\section{Availability of data and materials}

Please contact the corresponding author for reasonable data requests.

\section{Competing interests}

The authors declare that they have no competing interests.

\section{Funding}

This work was supported by the Ministry of Education, Culture, Sports, Science, and Technology, Japan (grant numbers 18K07406 [K. Tsunekawa] and 17H04109 [M. Murakami]).

\section{Authors' contributions}


KT participated in the collection and analysis of data and writing of the manuscript. RM, LM, KU, YS, YY, $\mathrm{HI}, \mathrm{AY}, \mathrm{OA}, \mathrm{NK}$, and TK participated in data collection and analysis. MM participated in conception of the study, supervision, and manuscript editing. All authors read and approved the final manuscript.

\section{Acknowledgments}

We thank Mayumi Nishiyama for their technical assistance and helpful discussion.

\section{References}

1. Suryawan A, Hawes JW, Harris RA, Shimomura Y, Jenkins AE, Hutson SM. Molecular model of human branched-chain amino acid metabolism. Am J Clin Nutr. 1998;68:72-81.

2. Zhang SH, Zeng XF, Ren M, Mao XB, Qiao SY. Novel metabolic and physiological functions of branched chain amino acids: a review. J Anim Sci Biotechnol. 2017;8:10

3. Anthony JC, Yoshizawa F, Anthony TG, Vary TC, Jefferson LS, Kimball SR. Leucine stimulates translation initiation in skeletal muscle of postabsorptive rats via a rapamycin-sensitive pathway. $J$ Nutr. 2000;130:2413-19.

4. Katsanos CS, Kobayashi H, Sheffield-Moore M, Aarsland A, Wolfe RR. A high proportion of leucine is required for optimal stimulation of the rate of muscle protein synthesis by essential amino acids in the elderly. Am J Physiol Endocrinol Metab. 2006;291:E381-7.

5. Martin L, Birdsell L, MacDonald N, Reiman T, Clandinin MT, McCargar LJ, et al. Cancer cachexia in the age of obesity: Skeletal muscle depletion is a powerful prognostic factor, independent of body mass index. J Clin Oncol. 2013;31:1539-47.

6. Landi F, Liperoti R, Russo A, Giovannini S, Tosato M, Capoluongo E, et al. Sarcopenia as a risk factor for falls in elderly individuals: Results from the ilSIRENTE study. Clin Nutr. 2012;31:652-58.

7. Tsuji S, Koyama S, Taniguchi R, Fujiwara T, Fujiwara H, Sato Y. Nutritional status of outpatients with chronic stable heart failure based on serum amino acid concentration. J Cardiol. 2018;72:458-65.

8. Nishikawa $\mathrm{H}$, Enomoto $\mathrm{H}$, Ishii $\mathrm{A}$, Iwata $\mathrm{Y}$, Miyamoto $\mathrm{Y}$, Ishii $\mathrm{N}$, et al. Development of a simple predictive model for decreased skeletal muscle mass in patients with compensated chronic liver disease. Hepatol Res. 2017;47:1223-34.

9. Shimomura $Y$, Kobayashi H, Mawatari K, Akita K, Inaguma A, Watanabe S, et al. Effects of squat exercise and branched-chain amino acid supplementation on plasma free amino acid concentrations in young women. J Nutr Sci Vitaminol (Tokyo). 2009;55:288-91.

10. Reidy PT, Rasmussen BB. Role of ingested amino acids and protein in the promotion of resistance exercise-induced muscle protein anabolism. J Nutr. 2016;146:155-83.

11. Maclean DA, Graham TE, Saltin B. Branched-chain amino acids augment ammonia metabolism while attenuating protein breakdown during exercise. Am J Physiol Endocrinol Metab. 
1994;267:E1010-22.

12. Howatson G, Hoad M, Goodall S, Tallent J, Bell PG, French DN. Exercise-induced muscle damage is reduced in resistance-trained males by branched chain amino acids: a randomized, double-blind, placebo controlled study. J Int Soc Sports Nut. 2012;9:20.

13. Kersten S. Physiological regulation of lipoprotein lipase. Biochim Biophys Acta. 2014; 1841:913-33.

14. Fong LG, Young SG, Beigneux AP, Bensadoun A, Oberer M, Jiang H, et al. GPIHBP1 and plasma triglyceride metabolism. Trends Endocrinol Metab. 2016;27:455-69.

15. Matsumoto R, Tsunekawa K, Shoho Y, Yanagawa Y, Kotajima N, Matsumoto S, et al. Association between skeletal muscle mass and serum concentrations of lipoprotein lipase, GPIHBP1, and hepatic triglyceride lipase in young Japanese men. Lipids in Health Dis. 2019;18:84.

16. Salvatore D, Simonides WS, Dentice M, Zavacki AM, Larsen PR. Thyroid hormones and skeletal muscle-new insights and potential implications. Nat Rev Endocrinol. 2014;10:206-14.

17. Bloise FF, Cordeiro A, Ortiga-Carvalho TM. Role of thyroid hormone in skeletal muscle physiology. $\mathrm{J}$ Endocrinol. 2018;236:R57-68.

18. Shimbo K, Yahashi A, Hirayama K, Nakazawa M, Miyano H. Multifunctional and highly sensitive precolumn reagents for amino acids in liquid chromatography/tandem mass spectrometry. Anal Chem. 2009;81:5172-79.

19. Lippi G, Brocco G, Franchini M, Schena F, Guidi G. Comparison of serum creatinine, uric acid, albumin and glucose in male professional endurance athletes compared with healthy controls. Clin Chem Lab Med. 2004;42:644-47.

20. Desgorces FD, Moinard C, Chennaoui M, Toussaint JF, Petibois C, Noirez P. Development of a specific index to detect malnutrition in athletes: Validity in weight class or intermittent fasted athletes. Biochim Open. 2016;4:1-7.

21. Crespo R, Relea P, Lozano D, MacarroSanchez M, Usabiaga J, Villa LF, et al. Biochemical markers of nutrition in elite-marathon runners. J Sports Med Phys Fit. 1995;35:268-72.

22. Moon MK, Lee YJ, Choi SH, Lim S, Yang EJ, Lim JY, et al. Subclinical hypothyroidism has little influences on muscle mass or strength in elderly people. J Korean Med Sci. 2010;25:1176-81.

23. Sheng $Y$, Ma D, Zhou Q, Wang L, Sun M, Wang S, et al. Association of thyroid function with sarcopenia in elderly Chinese euthyroid subjects. Aging Clin Exp Res. 2019;31:1113-20.

24. Fidale TM, Antunes HKM, Roever L, Goncalves A, Puga GM, Silva RPM, et al. Leucine supplementation improves effort tolerance of rats with hyperthyroidism. Front Physiol. 2018;9:1632.

25. Zhang Y, Kobayashi H, Mawatari K, Sato J, Bajotto G, Kitaura Y, et al. Effects of branched-chain amino acid supplementation on plasma concentrations of free amino acids, insulin, and energy substrates in young men. J Nutr Sci Vitaminol (Tokyo). 2011;57:114-17.

\section{Tables}

Table 1

Page $11 / 16$ 
Comparison of clinical characteristics between the wrestling athletes and the controls.

\begin{tabular}{|c|c|c|c|c|c|c|c|}
\hline & \multicolumn{2}{|c|}{$\begin{array}{l}\text { All participants } \\
\qquad(N=111)\end{array}$} & \multicolumn{2}{|c|}{$\begin{array}{l}\text { Control participants } \\
\qquad(N=41)\end{array}$} & \multicolumn{2}{|c|}{$\begin{array}{l}\text { Wrestling athletes } \\
\qquad(N=70)\end{array}$} & $p$ \\
\hline$\overline{\text { Weigh }}$ & 66.6 & $(62.3-74.2)$ & 64.2 & $(58.1-68.4)$ & 68.4 & $(63.6-75.9)$ & 0.012 \\
\hline $\operatorname{BMI}\left(\mathrm{kg} / \mathrm{m}^{2}\right)$ & 23.7 & $2.2-2$ & 21.7 & .1 & 24.2 & $-26.0)$ & $<0.001$ \\
\hline SMM (\%) & 3.5 & 9) & 30.5 & ) & 34.6 & $(32.4-39.0)$ & $<0.001$ \\
\hline $\mathrm{SMI}(\mathrm{kg} / \mathrm{r}$ & 11.9 & 1.04 & 10.5 & $(9.9-11.5)$ & 12.4 & $(11.9-13.0)$ & $<0.001$ \\
\hline FM (\%) & 7.8 & 165 & 9.1 & (6 & 7.3 & $(6.4-9.4)$ & $<0.001$ \\
\hline $\mathrm{FMI}\left(\mathrm{kg} / \mathrm{m}^{2}\right)$ & 2.7 & 1 & 3.2 & $(2.3-4.9)$ & 2.6 & $(2.2-3.5)$ & 24 \\
\hline Albumin $(\mathrm{g} / \mathrm{dL})$ & 4.5 & 1 & 4.8 & $(4.7-5.0)$ & 4.3 & $(4.2-4.5)$ & $<0.001$ \\
\hline Transthyretin ( $r$ & 29.8 & $(26.5-33.0)$ & 29.8 & $(25.3-32.2)$ & 29.8 & $(27.1-33.1)$ & 0.790 \\
\hline $\mathrm{RBP}(\mathrm{mg} / \mathrm{dL})$ & 3.0 & $(2.6-3.4)$ & 2.8 & $(2.5-3.3)$ & 3.1 & $(2.7-3.6)$ & 0.009 \\
\hline Transferrin (mg/dL) & 248 & $(228-268)$ & 255 & $(235-272)$ & 247 & $(222-264)$ & 0.071 \\
\hline $\mathrm{FT}_{3}(\mathrm{pg} / \mathrm{mL})$ & 3.22 & $(3.09-3.39)$ & 3.18 & $3.04-3.34)$ & 3.23 & $(3.10-3.41)$ & 0.115 \\
\hline $\mathrm{FT}_{4}(\mathrm{ng} / \mathrm{dL})$ & 1.04 & $(0.98-1.10)$ & 1.02 & $(0.98-1.10)$ & 1.05 & $(0.97-1.11)$ & 0.318 \\
\hline $\mathrm{TSH}(\mathrm{mlU} / \mathrm{ml}$ & 1.78 & $(1.24-2.25)$ & 1.84 & $(1.19-2.12)$ & 1.67 & $(1.36-2.30)$ & 0.647 \\
\hline BTR & 6.49 & $(5.80-7.58)$ & 7.62 & $(6.83-8.61)$ & 6.07 & $(5.51-6.64)$ & $<0.001$ \\
\hline
\end{tabular}

Data are expressed as median (25th-75th percentile).

Mann-Whitney $U$ test were used to compare the wrestling athletes with the control participants.

BMI, body mass index; SMM, weight-adjusted skeletal muscle mass; SMI, skeletal muscle index; FM, weight-adjusted fat mass; $\mathrm{FMl}$, fat mass index; RBP, retinol binding protein; $\mathrm{FT}_{3}$, free 3,5,3'-

triiodothyronine; $\mathrm{FT}_{4}$, free thyroxine; $\mathrm{TSH}$, thyrotropin; $\mathrm{BTR}$, branched-chain amino acid to tyrosine ratio.

\section{Table 2}

Spearman's correlation analyses between serum albumin concentration or BTR and clinical variables $(N=$ 111) 


\begin{tabular}{|c|c|c|c|c|}
\hline \multirow[b]{2}{*}{ Variable } & \multicolumn{2}{|c|}{ Albumin } & \multicolumn{2}{|c|}{ BTR } \\
\hline & $\rho$ & $p$ & $\rho$ & $p$ \\
\hline Weight (kg) & -0.213 & 0.025 & -0.214 & 0.024 \\
\hline $\mathrm{BMI}\left(\mathrm{kg} / \mathrm{m}^{2}\right)$ & -0.370 & $<0.001$ & -0.217 & 0.022 \\
\hline SMM (\%) & -0.315 & 0.001 & -0.424 & $<0.001$ \\
\hline $\operatorname{SMI}\left(\mathrm{kg} / \mathrm{m}^{2}\right)$ & -0.511 & $<0.001$ & -0.436 & $<0.001$ \\
\hline FM (\%) & 0.240 & 0.011 & 0.334 & $<0.001$ \\
\hline $\mathrm{FMI}\left(\mathrm{kg} / \mathrm{m}^{2}\right)$ & 0.104 & 0.279 & 0.238 & 0.012 \\
\hline Transthyretin (mg/dL) & 0.281 & 0.003 & 0.172 & 0.072 \\
\hline $\mathrm{RBP}(\mathrm{mg} / \mathrm{dL})$ & 0.094 & 0.327 & -0.020 & 0.838 \\
\hline Transferrin $(\mathrm{mg} / \mathrm{dL})$ & 0.299 & 0.001 & 0.078 & 0.418 \\
\hline $\mathrm{FT}_{3}(\mathrm{pg} / \mathrm{mL})$ & 0.013 & 0.896 & -0.308 & 0.001 \\
\hline $\mathrm{FT}_{4}(\mathrm{ng} / \mathrm{dL})$ & 0.107 & 0.262 & -0.077 & 0.421 \\
\hline $\mathrm{TSH}(\mathrm{mlU} / \mathrm{mL})$ & 0.123 & 0.199 & -0.019 & 0.846 \\
\hline BTR & 0.438 & $<0.001$ & & \\
\hline
\end{tabular}

BMI, body mass index; SMM, weight-adjusted skeletal muscle mass; SMI, skeletal muscle index; FM, weight-adjusted fat mass; FMI, fat mass index; RBP, retinol binding protein; $\mathrm{FT}_{3}$, free 3,5,3'triiodothyronine; $\mathrm{FT}_{4}$, free thyroxine; $\mathrm{TSH}$, thyrotropin; $\mathrm{BTR}$, branched-chain amino acid to tyrosine ratio.

\section{Figures}




\section{Figure 1}

A
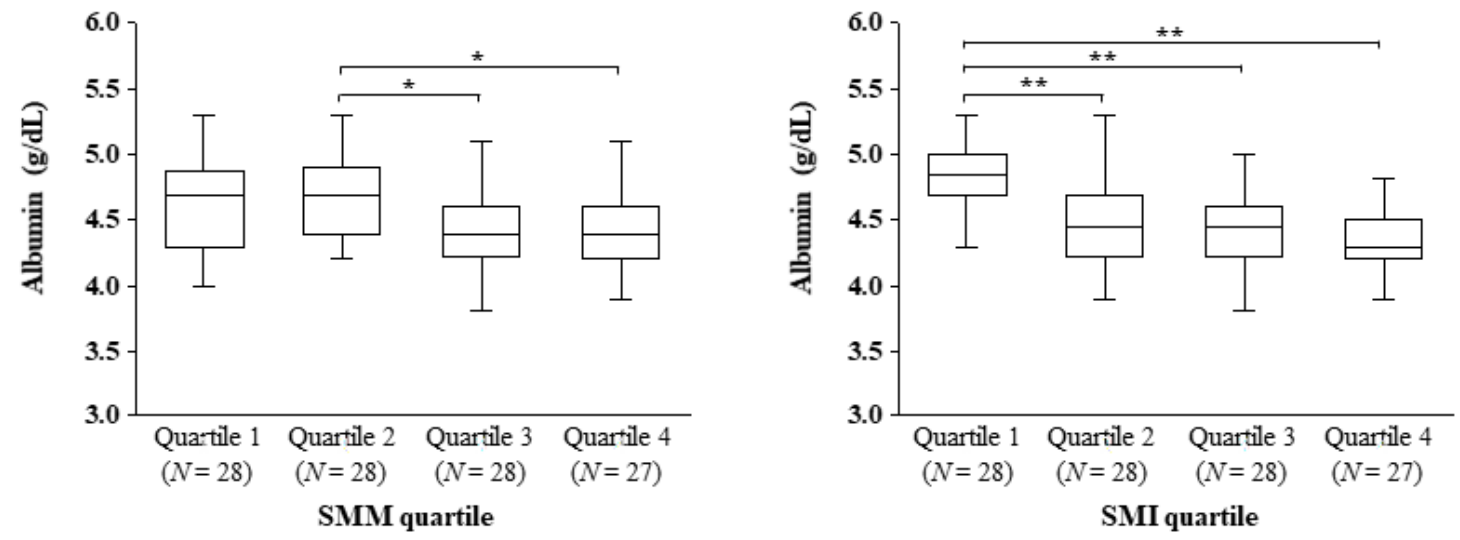

B
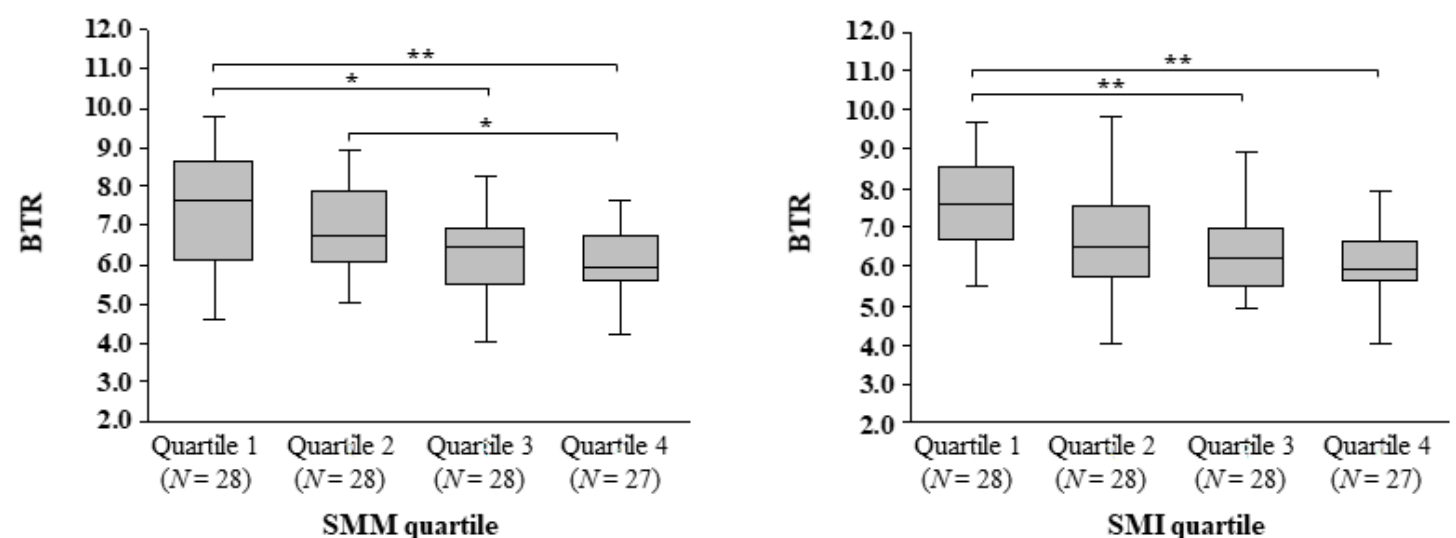

Figure 1

Association between skeletal muscle mass and serum albumin concentration or BTR ( $N=111$ men). Comparisons of (A) serum albumin concentration and (B) branched-chain amino acids to tyrosine ratio (BTR) between the quartiles for weight-adjusted skeletal muscle mass (SMM) and skeletal muscle index (SMI). The SMM quartiles were as follows: Quartile 1, SMM $\leq 48.0 \%$; Quartile 2, 48.0\% < SMM $\leq 50.0 \%$; Quartile 3, 50.0\% < SMM $\leq 51.9 \%$; and Quartile 4, SMM > 51.9\%. The SMI quartiles were as follows: Quartile 1, SMI $\leq 11.0 \mathrm{~kg} / \mathrm{m} 2$; Quartile 2, $11.0<\mathrm{SMl} \leq 11.9 \mathrm{~kg} / \mathrm{m} 2$; Quartile 3, $11.9<\mathrm{SMl} \leq 12.5 \mathrm{~kg} / \mathrm{m} 2$; and Quartile 4, SMI $>12.5 \mathrm{~kg} / \mathrm{m} 2$. The groups were compared with Kruskal-Wallis tests and Bonferroni multiple comparison tests $\left({ }^{\star} p<0.05\right.$, $\left.{ }^{*} \mathrm{p}<0.01\right)$. 
Figure 2

A
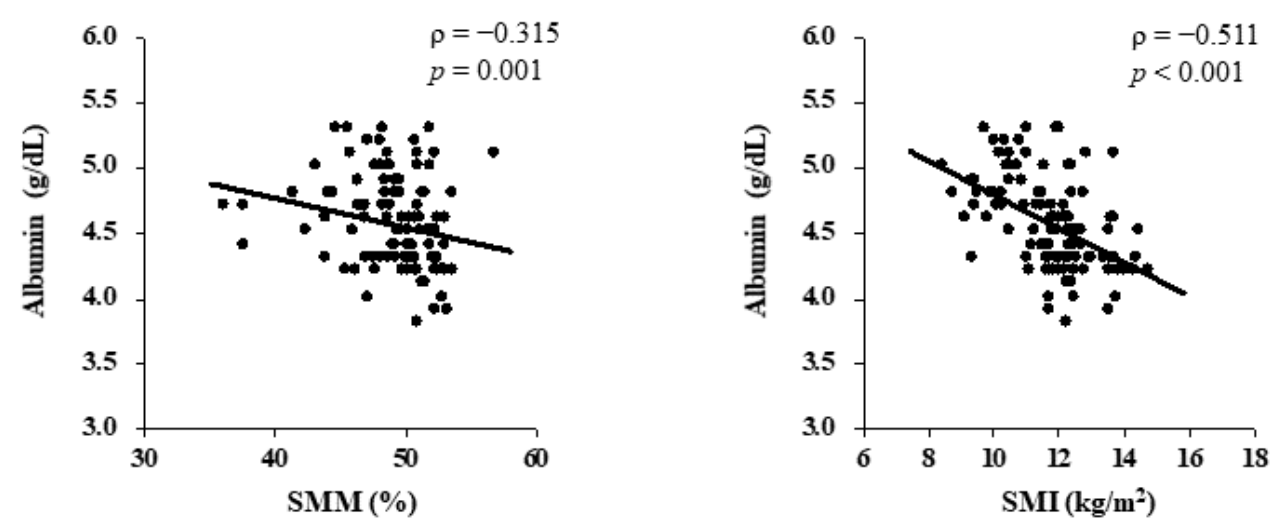

B
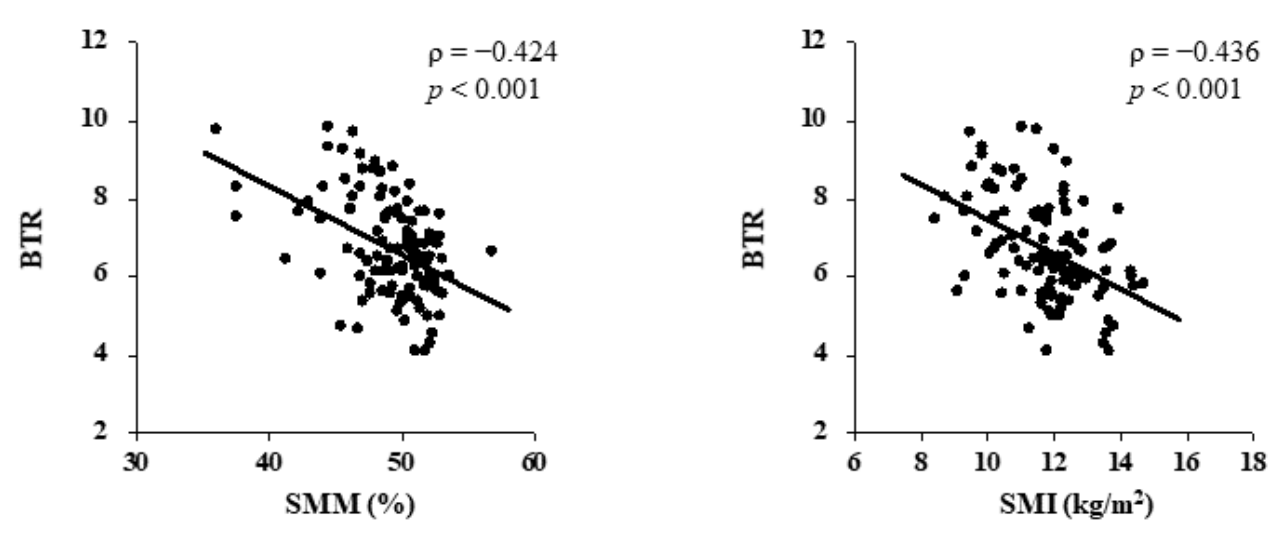

Figure 2

Correlations between skeletal muscle mass and serum albumin concentration or BTR ( $\mathrm{N}=111 \mathrm{men})$. Correlations between SMM or SMM and (A) serum albumin concentration or (B) BTR. The correlations were evaluated using Spearman's correlation analysis. 
Figure 3

A

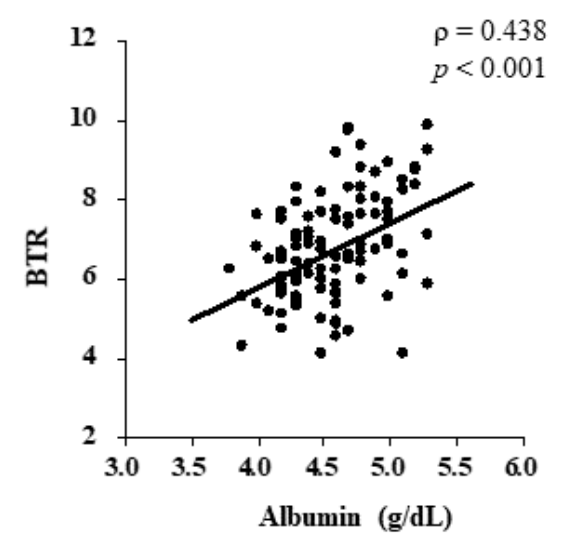

B

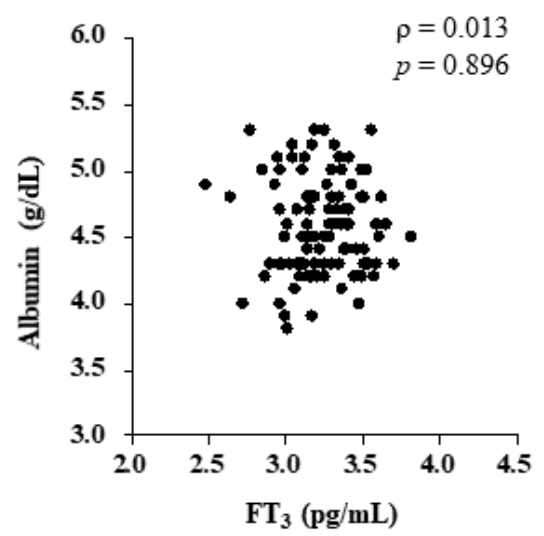

C

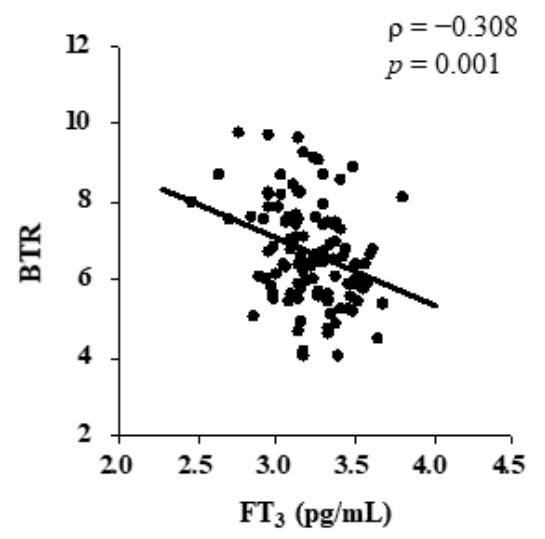

Figure 3

Correlations among serum albumin, BTR, and FT3 ( $\mathrm{N}=111$ men). Results of the Spearman's correlation analyses $(A)$ between serum albumin concentration and BTR, $(B)$ between serum albumin concentration and free 3,5,3'-triiodothyronine (FT3), and (C) between BTR and FT3. 